\title{
OSCILLATION RESULTS ON LINEAR SYSTEMS OF DIFFERENCE EQUATIONS
}

\author{
QINGKAI KONG
}

(Communicated by Hal L. Smith)

\begin{abstract}
We consider the difference system $a_{n+1}-a_{n}+\sum_{i=1}^{m} Q_{i} a_{n-k_{i}}=0$ where $k_{i} \in \mathbb{Z}, Q_{i} \in \mathbb{R}^{r \times r}, i=1, \ldots, m$, and systems of more general forms. We establish explicit conditions for the systems to be oscillatory. Those conditions are necessary and sufficient for special cases.
\end{abstract}

\section{INTRODUCTION AND PRELIMINARIES}

We consider oscillation problems for the linear vector difference equations of the type

$$
a_{n+k}+P_{1} a_{n+k-1}+\cdots+P_{k} a_{n}=0, \quad n=1,2, \ldots,
$$

where $k \in \mathbb{N}=\{1,2, \ldots\}, P_{i} \in \mathbb{R}^{r \times r}$, the space of all $r \times r$ matrices, $i=$ $1,2, \ldots, k$. By a solution of Eq. (1.1) we mean a sequence $\left\{a_{n}\right\}$ of points $a_{n} \in$ $\mathbb{R}^{r}$ for $n=1,2, \ldots$ which satisfies Eq. (1.1). As customary, a sequence of real numbers $\left\{x_{n}\right\}$ is said to be oscillatory if the terms $x_{n}$ are not eventually positive or eventually negative; a solution $\left\{a_{n}\right\}$ of Eq. (1.1) is said to be oscillatory if every component of $\left\{a_{n}\right\}$ is oscillatory; Eq. (1.1) is said to be oscillatory if all its solutions are oscillatory.

The following system of difference equations can be regarded as a special form of Eq. (1.1) if the leading coefficient is nonsingular:

$$
a_{n+1}-a_{n}+\sum_{i=1}^{m} Q_{i} a_{n-k_{i}}=0, \quad n=1,2, \ldots,
$$

where $k_{i} \in \mathbb{Z}=\{0, \pm 1, \pm 2, \ldots\}, Q_{i} \in \mathbb{R}^{r \times r}, i=1,2, \ldots, m$. One may think of Eq. (1.2) as being a discrete analogue of the differential equation

$$
\dot{y}(t)+\sum_{i=1}^{m} Q_{i} y\left(t-\tau_{i}\right)=0 .
$$

By analogy with Eq. (1.3), Eq. (1.2) is said to be of the delay, advanced, or mixed type if the $k_{i}$ 's are all positive, all negative, or otherwise, respectively.

Received by the editors March 16, 1994.

1991 Mathematics Subject Classification. Primary 39A10, 39A12.

Key words and phrases. Vector difference systems, linear, autonomous, oscillation theory.

(C) 1995 American Mathematical Society 
Discussion on Eq. (1.2) for the scalar case has had an extensive treatment in the literature; see, e.g. $[3,5,6,7,9]$ and the references therein. However, to the best of the author's knowledge, the oscillation theory for the vector equations (1.1) and (1.2) has not yet been well developed. In fact, the main aspect of oscillation in systems of this type is the following result for Eq. (1.2) (a similar result for Eq. (1.1) is also obtained), which is an analogue to the result in [1] for differential equation (1.3).

Lemma 1.1 [6,7]. Assume $k_{i} \in \mathbb{Z}$ and $Q_{i} \in \mathbb{R}^{r \times r}, i=1, \ldots, m$. Then Eq. (1.2) is oscillatory if and only if its characteristic equation

$$
\operatorname{det}\left[(\sigma-1) I+\sum_{i=1}^{m} Q_{i} \sigma^{-k_{i}}\right]=0
$$

has no positive roots.

Since this condition is very difficult to apply, it is posed in $[6$, p. 196] as an open problem to obtain explicit conditions in terms of the $Q_{i}$ 's for oscillation of Eq. (1.2). In the present paper, this problem is solved by employing Lozenskii measures of matrices. Furthermore, necessary and sufficient conditions of explicit forms are also obtained for special cases.

In order to establish our oscillation criteria, we introduce the following definitions and notation by [2].

For any $A \in \mathbb{R}^{r \times r}$ we denote by $\lambda_{i}(A), i=1, \ldots, r$, the eigenvalues of $A$ satisfying

$$
\operatorname{Re} \lambda_{1}(A) \geq \operatorname{Re} \lambda_{2}(A) \geq \cdots \geq \operatorname{Re} \lambda_{r}(A) .
$$

Definition 1.1. We define $\|A\|_{i}=\sup _{x \in \mathbb{R}^{n}, x \neq 0}\|A x\|_{i} /\|x\|_{i}, i=1,2, \ldots, \infty$, where $x=\left\{x_{1}, \ldots, x_{r}\right\}^{T}$,

$$
\|x\|_{i}=\left(\sum_{j=1}^{r}\left|x_{j}\right|^{i}\right)^{1 / i}, \quad i<\infty, \quad \text { and } \quad\|x\|_{\infty}=\max _{1 \leq j \leq r}\left\{\left|x_{j}\right|\right\} .
$$

The Lozenskii measures $\mu_{i}(A), \nu_{i}(A), i=1,2, \ldots, \infty$, are defined by

$$
\mu_{i}(A)=\lim _{h \rightarrow 0^{+}} \frac{\|I+h A\|_{i}-1}{h} \text { and } \nu_{i}(A)=-\mu_{i}(-A) .
$$

In general, without specification, we denote by $\mu(A)$ and $\nu(A)$ any pair of $\mu_{i}(A)$ and $\nu_{i}(A), i=1,2, \ldots, \infty$. It has been shown that $\mu_{i}(A)$ and $\nu_{i}(A)$, $i=1,2 \ldots, \infty$, exist for any $r \times r$ matrix $A$ and can be explicitly evaluated for $i=1,2, \infty$ :

$$
\begin{array}{ll}
\mu_{1}(A)=\sup _{j}\left\{a_{j j}+\sum_{i, i \neq j}\left|a_{i j}\right|\right\}, & \nu_{1}(A)=\inf _{j}\left\{a_{j j}-\sum_{i, i \neq j}\left|a_{i j}\right|\right\} ; \\
\mu_{2}(A)=\lambda_{1}\left(\frac{1}{2}\left(A+A^{T}\right)\right), & \nu_{2}(A)=\lambda_{r}\left(\frac{1}{2}\left(A+A^{T}\right)\right) ; \\
\mu_{\infty}(A)=\sup _{i}\left\{a_{i i}+\sum_{j, j \neq i}\left|a_{i j}\right|\right\}, & \nu_{\infty}(A)=\inf _{i}\left\{a_{i i}-\sum_{j, j \neq i}\left|a_{i j}\right|\right\} .
\end{array}
$$


The following properties are known: for any $r \times r$ matrices $A$ and $B$ and any Lozenskii measures $\mu$ and $\nu$ we have

(i) $\mu(A+B) \leq \mu(A)+\mu(B), \nu(A+B) \geq \nu(A)+\nu(B)$;

(ii) $\mu(\alpha A)=\alpha \mu(A), \nu(\alpha A)=\alpha \nu(A)$, for $\alpha>0$;

(iii) $\mu(A) \geq \operatorname{Re} \lambda_{1}(A), \nu(A) \leq \operatorname{Re} \lambda_{r}(A)$;

(iv) $\mu(I)=1$ where $I$ is the $r \times r$ identity matrix.

From (iii) we can see that if $\mu(A)<0$ or $\nu(A)>0$, then $\operatorname{det} A \neq 0$.

Furthermore, for any $A, C \in \mathbb{R}^{r \times r}$ with $\mu(C) \neq 0$, we denote

$$
\begin{aligned}
& \mu_{C}^{+}(A)=\frac{\mu(A C)}{\mu(C)} \quad \text { and } \quad \mu_{C}^{-}(A)=\frac{\mu(C A)}{\mu(C)} ; \\
& \nu_{C}^{+}(A)=\frac{\nu(A C)}{\mu(C)} \quad \text { and } \quad \nu_{C}^{-}(A)=\frac{\nu(C A)}{\mu(C)} .
\end{aligned}
$$

In general, we denote by $\mu_{C}(A)$ or $\nu_{C}(A)$ any one of $\mu_{C}^{ \pm}(A)$ or any one of $\nu_{C}^{ \pm}(A)$, respectively, and call them $C$-weighted Lozenskii measures. In most cases, $\mu_{C}(A) \neq \mu(A)$ and $\nu_{C}(A) \neq \nu(A)$. For example, let $\nu=\nu_{1}$ and $C=$ $\operatorname{diag}\left\{c_{1}, \ldots, c_{r}\right\}$ with $\max _{1 \leq i \leq r}\left\{c_{i}\right\}=1$. Then

$$
\nu_{C}^{+}(A)=\inf _{j}\left\{c_{j} a_{j j}-\sum_{i, i \neq j}\left|c_{j} a_{i j}\right|\right\}, \quad \nu_{C}^{-}(A)=\inf _{j}\left\{c_{j} a_{j j}-\sum_{i, i \neq j}\left|c_{i} a_{i j}\right|\right\} .
$$

However, for the case that $r=1, \mu_{C}(A)=\nu_{C}(A)=A$ for any $C \neq 0$.

\section{MAIN Results}

In this section we first state some oscillation criteria for Eq. (1.2), then extend them to the general equation (1.1). All the proofs are left to the last section. Throughout this section we use the convention that $0^{0}=1$.

In the first two theorems we obtain results for Eq. (1.2) of the delay and advanced types, respectively.

Theorem 2.1. Let $k_{i} \in \mathbb{N}$ and $Q_{i} \in \mathbb{R}^{r \times r}, i=1, \ldots, m$. Assume there exists $C \in \mathbb{R}^{r \times r}$ satisfying $\nu(C)>0, \nu_{C}\left(Q_{i}\right) \geq 0, i=1, \ldots, m$. Then Eq. (1.2) is oscillatory if either one of the following holds:

(i) $\sum_{i=1}^{m} \nu_{C}\left(Q_{i}\right) \frac{\left(k_{i}+1\right)^{k_{i}+1}}{k_{i}^{k_{i}}}\left\{\begin{array}{ll}>1, & \text { if } m=1 \\ \geq 1, & \text { if } m>1\end{array}\right.$; or

(ii) $m\left(\prod_{i=1}^{m} \nu_{C}\left(Q_{i}\right)\right)^{1 / m} \frac{(k+1)^{k+1}}{k^{k}}>1, \quad$ where $k=\frac{1}{m} \sum_{i=1}^{m} k_{i}$.

Theorem 2.2. Let $k_{i} \in\{-1,-2, \ldots\}$ and $Q_{i} \in \mathbb{R}^{r \times r}, i=1, \ldots, m$. Assume there exists $C \in \mathbb{R}^{r \times r}$ satisfying $\nu(C)>0, \mu_{C}\left(Q_{i}\right) \leq 0, i=1, \ldots, m$. Then $E q$. (1.2) is oscillatory if either one of the following holds:

(i) $\sum_{i=1}^{m} \mu_{C}\left(Q_{i}\right) \frac{\left(k_{i}+1\right)^{k_{i}+1}}{k_{i}^{k_{i}}}\left\{\begin{array}{ll}>1, & \text { if } m=1 \\ \geq 1, & \text { if } m>1\end{array}\right.$; or

(ii) $m\left(\prod_{i=1}^{m}\left|\mu_{C}\left(Q_{i}\right)\right|\right)^{1 / m}\left|\frac{(k+1)^{k+1}}{k^{k}}\right|>1$, where $k=\frac{1}{m} \sum_{i=1}^{m} k_{i}$. 
Remark 2.1. (i) For the scalar equation corresponding to Eq. (1.2), noting that $\mu_{C}\left(Q_{i}\right)=\nu_{C}\left(Q_{i}\right)=Q_{i}$ for any $C>0$, the above theorems cover and even improve previous results (see for example, Theorems 7.3.1 and 7.3.2 in [6]), since the equalities in condition (i) of both theorems are allowed.

(ii) For the general case, if we choose $C=I$, then the criteria are given in terms of the regular Lozenskii measures, and hence are analogues to those for systems of differential equations; see $[4,8]$.

(iii) The conditions are substantially improved by introducing the weight matrix $C$ in the Lozenskii measures. To see the significant role played by $C$, look at the following examples.

Example 2.1. Consider the equation

$$
a_{n+1}-a_{n}+Q a_{n-1}=0, \quad n=1,2, \ldots,
$$

where $Q=\left[\begin{array}{cc}1 & 2 \\ -2 & 7\end{array}\right]$. Since $\nu_{1}(Q)=-1<0$, Theorem 2.1 does not work for Eq. (2.2) with regular Lozenskii measures. However, if we choose $C=\operatorname{diag}\left\{1, \frac{1}{3}\right\}$, then the corresponding $C$-weighted Lozenskii measure of $Q$ is

$$
\nu_{C}^{-}(Q)=\frac{\nu_{1}(C Q)}{\mu_{1}(C)}=\nu_{1}\left(\left[\begin{array}{cc}
1 & 2 \\
-\frac{2}{3} & \frac{7}{3}
\end{array}\right]\right)=\frac{1}{3} .
$$

It is easy to see that $(2.1)$ is satisfied with $\nu_{C}^{-}(Q)$. By Theorem 2.1, Eq. (2.2) is oscillatory.

Example 2.2. Consider Eq. (2.2) with $Q=\frac{1}{4}\left[\begin{array}{cc}2 & 1 \\ -1 & 3\end{array}\right]$. Since $\nu_{1}(Q)=\frac{1}{4},(2.1)$ does not hold for $\nu_{1}(Q)$. However, if we choose $C=\frac{1}{5}\left[\begin{array}{cc}4 & -1 \\ 1 & 2\end{array}\right]$, then the corresponding

$$
\nu_{C}^{+}(Q)=\frac{\nu_{1}(Q C)}{\mu_{1}(C)}=\nu_{1}\left(\frac{1}{20}\left[\begin{array}{cc}
9 & 0 \\
-1 & 7
\end{array}\right]\right)=\frac{7}{20},
$$

and (2.1) is satisfied with $\nu_{C}^{+}(Q)$. By Theorem (2.1), Eq. (2.2) is oscillatory.

Now we consider a special form of Eq. (1.2) when $m=1$ :

$$
a_{n+1}-a_{n}+Q a_{n-k}=0 \text {. }
$$

If $Q$ is a symmetric matrix, then some new necessary and sufficient conditions can be found for Eq. (2.3) to be oscillatory.

Theorem 2.3. Let $Q \in \mathbb{R}^{r \times r}$ be symmetric. Denote by $\lambda_{r}(Q)$ and $\lambda_{1}(Q)$ the least eigenvalue and the largest eigenvalue of $Q$, respectively. Then $E q .(2.3)$ is oscillatory if and only if one of the following holds:

(i) $k=0$ and $\lambda_{r}(Q) \geq 1$;

(ii) $k=-1$ and $\lambda_{1}(Q) \leq-1$;

(iii) $k \in\{1,2, \ldots\}$ and $\lambda_{r}(Q) \frac{(k+1)^{k+1}}{k^{k}}>1$;

(iv) $k \in\{-2,-3, \ldots\}$ and $\lambda_{1}(Q) \frac{(k+1)^{k+1}}{k^{k}}>1$.

Remark 2.2. (i) For the scalar equation corresponding to Eq. (2.3), noting that $\lambda_{r}(Q)=\lambda_{1}(Q)=Q$, the above theorem coincides with previous results; see for example, Theorem 7.2.1 in [6].

(ii) One cannot expect to extend Theorem 2.3 to the general case where $Q$ is nonsymmetric. This reveals the fact that Eq. (2.3) with symmetric $Q$ is much closer to the corresponding scalar equation in nature. 
(iii) The significance of Theorem 2.3 lies in that, different from Lemma 1.1, it presents explicit necessary and sufficient conditions, rather than implicit conditions given by transcendental equations, and hence is very easy to apply.

From Section 1, we know that for symmetric matrix $Q$ we have $\lambda_{1}(Q)=$ $\mu_{2}(Q)$ and $\lambda_{r}(Q)=\nu_{2}(Q)$. However, we cannot expect to improve the conditions in Theorem 2.3 by replacing $\lambda_{1}(Q)$ and $\lambda_{r}(Q)$ by other Lozenskii measures or by $C$-weighted Lozenskii measures, since all the conditions are necessary and sufficient. Therefore, as a by-product of Theorem 2.3, the following properties of Lozenskii measures are deduced, which are of some interest in their own right.

Corollary 2.1. Assume that $A, C \in \mathbb{R}^{r \times r}, A$ is symmetric and positive definite, and $\nu(C)>0$ for any pair of Lozenskii measures $\mu$ and $\nu$. Then

$$
\nu(A C) \leq \lambda_{r}(A) \mu(C) \text { and } \nu(C A) \leq \lambda_{r}(A) \mu(C) .
$$

The following example is an immediate result of Theorem 2.3.

Example 2.3. Consider Eq. (2.3) with $k \in\{1,2, \ldots\}$ and $Q=q\left[\begin{array}{cc}2 & \sqrt{2} \\ \sqrt{2} & 3\end{array}\right]$ for $q>0$. Since $\lambda_{2}(Q)=q$, by Theorem 2.3 , Eq. (2.3) is oscillatory if and only if $q \frac{(k+1)^{k+1}}{k^{k}}>1$.

At last, we extend the above criteria for Eq. (1.2) of the delay and advanced types to the general equation (1.1). Since Eq. (1.2) of the mixed type can be rewritten in the form (1.1), our results are also applicable to equations of this type.

Eq. (1.1) is equivalent to each of the following equations:

$$
\begin{aligned}
& a_{n+1}+Q_{0} a_{n}+Q_{1} a_{n-1}+\cdots+Q_{m} a_{n-m}=0 \\
& a_{n+1}-Q_{0} a_{n}+Q_{1} a_{n-1}+\cdots+Q_{m} a_{n-m}=0 .
\end{aligned}
$$

Two different generalizations of Theorem 2.1 to (2.4) and (2.5) are mentioned below. Similar generalizations of Theorem 2.2 can also be obtained.

Theorem 2.4. Let $Q_{i} \in \mathbb{R}^{r \times r}, i=0,1, \ldots, m$. Assume there exists $C \in \mathbb{R}^{r \times r}$ satisfying $\nu(C)>0, \nu_{C}\left(Q_{0}\right) \geq-1, \nu_{C}\left(Q_{i}\right) \geq 0, i=1, \ldots, m$. Then Eq. (2.4) is oscillatory if either one of the following holds:

$$
\begin{aligned}
& \text { (i) } \sum_{i=0}^{m} \nu_{C}\left(Q_{i}\right) \frac{(i+1)^{i+1}}{i^{i}} \geq 0 \text {, or } \\
& \text { (ii) }\left[\left(\nu_{C}\left(Q_{0}\right)+1\right) \prod_{i=1}^{m} \nu_{C}\left(Q_{i}\right)\right]^{1 / m} \frac{(m+2)^{\frac{m}{2}+1}}{2 m^{m / 2}}>1 .
\end{aligned}
$$

Theorem 2.5. Let $Q_{i} \in \mathbb{R}^{r \times r}, i=0,1, \ldots, m$. Assume there exists $C \in \mathbb{R}^{r \times r}$ satisfying $\nu(C)>0, \mu_{C}^{*}\left(Q_{0}\right)>0, \nu_{C}^{*}\left(Q_{i}\right) \geq 0, i=1, \ldots, m$, where

$$
\mu_{C}^{*}(A)=\mu_{C}(A) \mu(C) / \nu(C), \quad \nu_{C}^{*}(A)=\nu_{C}(A) \mu(C) / \nu(C) .
$$


Then Eq. (2.5) is oscillatory if either one of the following holds:
(i) $\sum_{i=1}^{m} \frac{\nu_{C}^{*}\left(Q_{i}\right)}{\left[\nu_{C}^{*}\left(Q_{0}\right)\right]^{i+1}} \frac{(i+1)^{i+1}}{i^{i}}\left\{\begin{array}{ll}>1, & \text { if } m=1 \\ \geq 1, & \text { if } m>1\end{array}\right.$;or
(ii) $m\left(\prod_{i=1}^{m} \nu_{C}^{*}\left(Q_{i}\right)\right)^{1 / m} \frac{(k+1)^{k+1}}{k^{k}}>\left[\nu_{C}^{*}\left(Q_{0}\right)\right]^{k+1}$.

\section{Proofs}

The following lemma is needed in the proofs and can be easily proved.

Lemma 3.1. Let $\alpha>0$ be a constant.

(i) For any $k \in\{0,1,2, \ldots\}, \inf _{\sigma \in(0, \alpha)} \frac{\sigma^{-k}}{\alpha-\sigma}=\frac{(k+1)^{k+1}}{k^{k} \alpha^{k+1}}$;

(ii) For any $k \in\{-1,-2, \ldots\}, \sup _{\sigma \in(\alpha, \infty)} \frac{\sigma^{-k}}{\alpha-\sigma}=\frac{(k+1)^{k+1}}{k^{k} \alpha^{k+1}}$.

In this section, all proofs are only given for the case that $\nu_{C}(A)=\nu_{C}^{-}(A)$ and $\mu_{C}(A)=\mu_{C}^{-}(A)$ when weighted Lozenskii measures are involved. The other case can be similarly proved and hence is omitted.

Proof of Theorem 2.2. Let

$$
G(\sigma)=(\sigma-1) I+\sum_{i=1}^{m} Q_{i} \sigma^{-k_{i}} \quad \text { and } \quad F(\sigma)=C G(\sigma) .
$$

Then for $\sigma \geq 1$

$$
\nu(F(\sigma)) \geq(\sigma-1) \nu(C)+\sum_{i=1}^{m} \nu\left(C Q_{i}\right) \sigma^{-k_{i}}>0,
$$

which implies that $\operatorname{det} F(\sigma) \neq 0$ for $\sigma \geq 1$. As a consequence, $\operatorname{det} G(\sigma) \neq 0$ for $\sigma \geq 1$. Assume $\sigma_{0} \in(0,1)$ is a solution of (1.4). Then

$$
\operatorname{det} F\left(\sigma_{0}\right)=(\operatorname{det} C)\left(\operatorname{det} G\left(\sigma_{0}\right)\right)=0 \text {, }
$$

which implies that $\nu\left(F\left(\sigma_{0}\right)\right) \leq 0$. Then by Lemma 3.1 (i) for $\alpha=1$

$$
\begin{aligned}
0 & \geq \frac{\nu\left(F\left(\sigma_{0}\right)\right)}{\left(1-\sigma_{0}\right) \mu(C)} \geq \frac{1}{\left(1-\sigma_{0}\right) \mu(C)}\left[\left(\sigma_{0}-1\right) \mu(C)+\sum_{i=1}^{m} \nu\left(C Q_{i}\right) \sigma_{0}^{-k_{i}}\right] \\
& =-1+\sum_{i=1}^{m} \nu_{C}\left(Q_{i}\right) \frac{\sigma_{0}^{-k_{i}}}{1-\sigma_{0}} \\
& \geq-1+\sum_{i=1}^{m} \nu_{C}\left(Q_{i}\right) \frac{\left(k_{i}+1\right)^{k_{i}+1}}{k_{i}^{k_{i}}}
\end{aligned}
$$

Noting that the equality in (3.3) cannot hold if $m>1$, we derive a contradiction to condition (i). 
Also, from (3.2) we have

$$
\begin{aligned}
1 & \geq m\left(\prod_{i=1}^{m} \nu_{C}\left(Q_{i}\right)\right)^{1 / m} \frac{\sigma_{0}^{-k}}{1-\sigma_{0}} \\
& \geq m\left(\prod_{i=1}^{m} \nu_{C}\left(Q_{i}\right)\right)^{1 / m} \frac{(k+1)^{k+1}}{k^{k}},
\end{aligned}
$$

contradicting condition (ii). By Lemma 1.1 Eq. (1.2) is oscillatory.

Proof of Theorem 2.2. Let $G(\sigma)$ and $F(\sigma)$ be defined by (3.1). For $\sigma \in(0,1]$,

$$
\mu(F(\sigma)) \leq(\sigma-1) \nu(C)+\sum_{i=1}^{m} \mu\left(C Q_{i}\right) \sigma^{-k_{i}}<0,
$$

which implies that $\operatorname{det} F(\sigma) \neq 0$, and hence $\operatorname{det} G(\sigma) \neq 0$ for $\sigma \in(0,1]$. Assume $\sigma_{0}>1$ is a solution of (1.4). Then $\operatorname{det} F\left(\sigma_{0}\right)=0$ implies that $\mu\left(F\left(\sigma_{0}\right)\right) \geq 0$. Then

$$
0 \leq \frac{\mu\left(F\left(\sigma_{0}\right)\right)}{\left(\sigma_{0}-1\right) \mu(C)} \leq 1+\sum_{i=1}^{m} \mu_{C}\left(Q_{i}\right) \frac{\sigma_{0}^{-k_{i}}}{\sigma_{0}-1} .
$$

The following is similar to that of Theorem 2.1 by applying Lemma 3.1 (ii) for $\alpha=1$.

Proof of Theorem 2.3. (i) For the case that $k=0$, let $G(\sigma)=(\sigma-1) I+Q$. Then

$$
\lambda_{r}(G(\sigma))=\sigma-1+\lambda_{r}(Q) .
$$

Since $\lambda_{r}(G(0))=-1+\lambda_{r}(Q)$ and $\lim _{\sigma \rightarrow+\infty} \lambda_{r}(G(\sigma))=+\infty$, we have that $\lambda_{r}(G(\sigma))>0$ for all $\sigma>0$ if and only if $\lambda_{r}(Q) \geq 1$. Noting that

$$
\operatorname{det} G(\sigma)=\prod_{i=1}^{r} \lambda_{i}(G(\sigma))
$$

and $\lambda_{i}(G(\sigma)) \geq \lambda_{r}(G(\sigma))$ for $i=1, \ldots, r$, we conclude that $\operatorname{det} G(\sigma) \neq 0$ for all $\sigma>0$ if and only if $\lambda_{r}(Q) \geq 1$.

(ii) Similar to (i).

(iii) Sufficiency is from Theorem 2.1 for $\nu=\nu_{2}$ and $C=I$. Now we prove the necessity. Assume

$$
\lambda_{r}(Q) \frac{(k+1)^{k+1}}{k^{k}} \leq 1 .
$$

Let $\sigma_{0}=\frac{k}{k+1}$. Then $\frac{\sigma_{0}^{-k}}{1-\sigma_{0}}=\frac{(k+1)^{k+1}}{k^{k}}$, and (3.5) becomes

or

$$
\lambda_{r}(Q) \frac{\sigma_{0}^{-k}}{1-\sigma_{0}} \leq 1
$$

$$
\sigma_{0}-1+\lambda_{r}(Q) \sigma_{0}^{-k} \leq 0
$$

Let $G(\sigma)=(\sigma-1) I+Q \sigma^{-k}$. Then

$$
\lambda_{r}\left(G\left(\sigma_{0}\right)\right)=\sigma_{0}-1+\lambda_{r}(Q) \sigma_{0}^{-k} \leq 0
$$


Choose $\sigma_{1}>1$ such that $\lambda_{r}\left(G\left(\sigma_{1}\right)\right)>0$. Then according to the continuity of $\lambda_{r}$, there exists $\sigma_{2} \in\left(\sigma_{0}, \sigma_{1}\right)$ such that $\lambda_{r}\left(G\left(\sigma_{2}\right)\right)=0$, and hence from (3.4), $\operatorname{det} G\left(\sigma_{2}\right)=0$. By Lemma 1.1 we conclude that Eq. (2.3) is not oscillatory.

(iv) Similar to (iii).

Proof of Theorem 2.4. Rewrite (2.4) into the form

$$
a_{n+1}-a_{n}+\left(Q_{0}+I\right) a_{n}+Q_{1} a_{n-1}+\cdots+Q_{m} a_{n-m}=0 \text {. }
$$

Since

$$
\nu_{C}\left(Q_{0}+I\right)=\nu_{C}\left(Q_{0}\right)+1,
$$

the conclusion follows immediately from Theorem 2.1.

Proof of Theorem 2.5. Let

$$
G(\sigma)=\sigma I-Q_{0}+\sum_{i=1}^{m} Q_{i} \sigma^{-i} \text { and } F(\sigma)=C G(\sigma) .
$$

Then for $\sigma \geq \mu\left(C Q_{0}\right) / \nu(C)$,

$$
\nu(F(\sigma)) \geq \sigma \nu(C)-\mu\left(C Q_{0}\right)+\sum_{i=1}^{m} \nu\left(C Q_{i}\right) \sigma^{-i}>0,
$$

which implies that $\operatorname{det} F(\sigma) \neq 0$, and hence $\operatorname{det} G(\sigma) \neq 0$ for

$$
\sigma \geq \mu\left(C Q_{0}\right) / \nu(C) .
$$

Assume $\sigma_{0} \in\left(0, \mu\left(C Q_{0}\right) / \nu(C)\right)$ is such that

$$
\operatorname{det} F\left(\sigma_{0}\right)=(\operatorname{det} C)\left(\operatorname{det} G\left(\sigma_{0}\right)\right)=0 .
$$

Then

$$
0 \geq \frac{\nu\left(F\left(\sigma_{0}\right)\right)}{\mu\left(C Q_{0}\right)-\sigma \nu(C)} \geq-1+\sum_{i=1}^{m} \nu_{C}^{*}\left(Q_{i}\right) \frac{\sigma^{-i}}{\mu_{C}^{*}\left(Q_{0}\right)-\sigma} .
$$

The rest of the proof is similar to that of Theorem 2.1 by using Lemma 3.1 for $\alpha=\mu_{C}^{*}\left(Q_{0}\right)$ and is omitted.

\section{REFERENCES}

1. O. Arino and I. Györi, Necessary and sufficient condition for oscillation of neutral differential system with several delays, J. Differential Equations 81 (1990), 98-105.

2. W. A. Copple, Stability and asymptotic behavior of differential equations, D. C. Heath and Company, Boston, MA, 1965.

3. R. D. Driver, G. Ladas, and P. N. Vlahos, Asymptotic behavior of a linear delay difference equation, Proc. Amer. Math. Soc. 115 (1992), 105-112.

4. L. Erbe and Q. Kong, Explicit conditions for oscillation of solutions to neutral differential systems, Hiroshima Math. J. 24 (1994), 317-329.

5. L. Erbe and B. G. Zhang, Oscillation of discrete analogues of delay equations, Differential Integral Equations 2 (1989), 300-309.

6. I. Györi and G. Ladas, Oscillation theory of delay differential equations with applications, Clarendon Press, Oxford, 1991.

7. I. Györi, G. Ladas, and L. Pakula, Conditions for oscillation of difference equations with applications to equations with piecewise constant arguments, SIAM J. Math. Anal. 22 (1991), 769-773. 
8. Q. Kong and H. I. Freedman, Oscillation in delay differential systems, Differential Integral Equations 6 (1993), 1325-1336.

9. G. Ladas, Explicit conditions for the oscillation of difference equations, J. Math. Anal. Appl. 153 (1990), 276-287.

Department of Mathematical Sciences, Northern Illinois University, DeKalb, ILLINOIS, 60115-2888

E-mail address: kong@math.niu.edu 